\title{
A Clinical Approach to Diagnosis and Treatment of Left Anterior Descending Artery Myocardial Bridge
}

\author{
Giuseppe Tarantini*, Luca Nai Fovino, Alberto Barioli, Alessandro Schiavo, Chiara Fraccaro \\ Department of Cardiac, Thoracic and Vascular Sciences, University of Padua, Padova, Italy
}

Article Info

\section{Article Notes}

Received: July 3, 2018

Accepted: September 5, 2018

\section{${ }^{*}$ Correspondence:}

Dr. Giuseppe Tarantini, Department of Cardiac, Thoracic and Vascular Sciences, University of Padova, Italy; Telephone No: $+390498211884$

(c) 2018 Tarantini G. This article is distributed under the terms of the Creative Commons Attribution 4.0 International License.

\section{Keywords}

Myocardial bridge

Left anterior descending artery

Functional assessment

\section{ABSTRACT}

The muscle overlying the intramyocardial segment of an epicardial coronary artery is defined as myocardial bridge (MB). The clinical relevance of MBs is extremely heterogeneous, ranging from incidental finding in asymptomatic patients to different clinical manifestations such as stable or unstable angina, arrhythmias, Takotsubo syndrome or other major cardiovascular events. Moreover, patients can evolve from "asymptomatic carriers" to "symptomatic carriers" over time. In this setting, haemodynamic assessment is challenging and optimal therapy still a matter of debate. This review summarizes epidemiology, pathophysiology, diagnostic work-up (including both morphological and functional assessment) and treatment of patients with MB involving the left anterior descending artery, suggesting a pragmatic clinical approach.

A coronary artery may dip into the myocardium for varying lengths, before reappearing on epicardial surface. The muscle overlying the intramyocardial segment of the coronary artery is termed myocardial bridge (MB), being the artery running within the myocardium referred to as tunneled artery. In $70 \%$ to $98 \%$ of cases, the MB involves the left anterior descending artery (LAD) ${ }^{1}$. Although MBs are reported to be present in about one-fourth of adults, their true prevalence varies widely according to the diagnostic method used to detect such an anatomic variant. In fact, pathological series reported higher rates of MBs compared to coronary angiography studies, in which MBs were detected as dynamic systolic compression of a coronary segment (milking effect). This mismatch is related to several factors including thickness and length of the MB, reciprocal orientation of coronary artery and myocardial fibers, intrinsic tone of the coronary artery wall, myocardial contractility and heart rate at the time of angiography, and finally observer experience ${ }^{2-4}$.

Although the presence of this congenital variant is generally considered a benign condition, patients with MB may present with silent ischemia, stable angina, acute coronary syndromes, Takotsubo cardiomyopathy, and malignant arrhythmias possibly leading to sudden cardiac death ${ }^{2,5-8}$. From a clinical point of view, it is important to understand why some MBs are (or become) symptomatic. In other words, when does a previously asymptomatic patient with a congenital MB become symptomatic? What additional factors unmask or aggravate a MB? Anatomical determinants that need to be taken into account are not only the depth and length of vessel encasement, but also the number of septal branches arising from or near the involved LAD segment. Moreover, pathophysiological factors that may unmask or exacerbate MBs are patient's age, heart rate, left ventricle (LV) hypertrophy, and the presence of coronary 
atherosclerosis, since all these may worsen the supplydemand mismatch imposed by the bridge, reducing coronary reserve ${ }^{9}$.

The compression of the tunneled segment of the bridged coronary artery may reduce not only the systolic but also the early hyperemic diastolic flow, due to tachycardia, delayed ventricular relaxation and phasic coronary spasm that prolongs also into diastole $\mathrm{e}^{10,11}$. Moreover, this pathophysiologic phenomenon causes ischemia by "intramural steal" or "branch steal" mechanism, which are linked to decreased perfusion pressure of septal branches and blood suction ${ }^{12,13}$. The disturbed flow pattern observed to the proximal end of the tunneled artery seems to be responsible of the formation and typical distribution of the coronary plaques, which are usually detected 20 to $30 \mathrm{~mm}$ proximal to the $\mathrm{MB}^{14,15}$. These altered biomechanical forces at the level of the MB could also lead to other potential complications, such as plaque vulnerability/thrombosis, increase in vasospasticity, and intimal injury that may further develop into coronary dissection ${ }^{16}$.

Morphological assessment of MBs can be performed with coronary angiography, obtained in different angiographic views to better visualize and characterize the tunneled artery, adjunctive intravascular imaging such as intravascular ultrasound (IVUS), and cardiac computed tomography (CCT). Coronary angiography typically reveals a dynamic systolic milking ( $\geq 50 \%$ systolic diameter stenosis not present or less evident in diastole) after intracoronary nitrates administration ${ }^{17,18}$. IVUS might improve the detection rate showing the systolic compression with delayed relaxation in diastole of the bridge segment, and the typical "half-moon phenomenon", an echolucent area surrounding the bridge and seen during the whole cardiac cycle. Moreover, IVUS is useful to better characterize the length, thickness, and location of the MB, as well as the presence, severity, and distribution of subangiographic atherosclerosis ${ }^{19}$. Finally, CCT is able to easily detect and characterize MBs, given its 3-dimensional capability and high spatial and contrast resolution. As a matter of fact, CCT can visualize the coronary lumen, the vessel wall, and the myocardial wall, hence allowing accurate definition of the MB's morphological features. The CCT scan protocol is not different from the conventional protocol used to assess coronary artery stenosis and post-processing software is helpful for the precise definition of MB's length and depth ${ }^{20}$.

Functional assessment of a bridged coronary artery might be hampered by several factors. Since stenoses in the context of MBs are not fixed and are dependent on the degree of extravascular compression and intramyocardial tension, their invasive assessment should not be limited to resting conditions but should include chronotropic and inotropic stimulation, in order to unmask their ischemic potential. Moreover, differently from fixed stenosis in which the difference between mean and diastolic pressure gradient values across the lesion are not significant, MB may cause significant diastolic pressure gradients but artificially negative systolic pressure gradients secondary to distal pressure overshooting ${ }^{21}$. Granted that, the traditional fractional flow reserve (FFR), a mean of systolic and diastolic pressure gradients, seems largely inadequate for the assessment of the hemodynamic significance of a $\mathrm{MB}$, because systolic pressure overshooting and negative systolic pressure gradients may lead to an underestimation of hemodynamic significance of the bridged segment $\left({ }^{21}\right)$. To this regard, diastolic FFR was proven to be more sensitive than conventional FFR for the investigation of $\mathrm{MBs}^{22}$.

Differently from FFR, the instantaneous wave-free ratio (iFR $®$, Volcano corporation, San Diego, CA, USA) is a lesionspecific, diastolic-only index, which might not be affected by the systolic pressure gradient inversion observed in significant MBs. In addition, the guidewire pull back allows anatomic mapping of the vessel to confirm the step-up at the level of $\mathrm{MB}^{23}$.

Our group recently analyzed a series of twenty patients with angina and abnormal non-invasive test suggestive of myocardial ischemia, and evidence of MB in the LAD without significant coronary artery disease at coronary angiogram. According to a prespecified protocol, all patients underwent functional evaluation of the MB with FFR and iFR, both at rest and after inotropic stimulus by intravenous dobutamine infusion, to unmask MB ischemic potential ${ }^{24}$. The FFR evaluation was negative at rest in all patients and, after inotropic infusion, when coronary compression was maximal and patients developed symptoms, median FFR did not significantly change compared to baseline ${ }^{24}$. This can be explained by the fact that, in the setting of inotropic stimulation, MBs can cause increase of diastolic but artificially negative systolic pressure gradients secondary to distal pressure overshooting. As previously stated, this phenomenon may produce an artificial elevation in the mean pressure used by traditional FFR (average systole and diastole), resulting in an underestimation of hemodynamic significance of the bridged LAD ${ }^{21,22}$. On the contrary, iFR at rest was abnormal in $65 \%$ of our patients with a clear stepup across the MB, as shown by iFR pullback during pressure wire pull back. After inotropic infusion, this index dropped in all cases. Despite the fact that iFR ischemic cut-off values after inotropic infusion are unknown, hemodynamic evaluation of MBs with iFR seems to be more consistent with patients' symptoms and noninvasive test results compared with $\mathrm{FFR}^{24}$ (figure 1).

Finally, measurement of intracoronary flow velocity and coronary flow reserve by the use of Doppler-tipped guidewires is another useful, but by far less diffused tool to evaluate MB's hemodynamics ${ }^{25}$. 

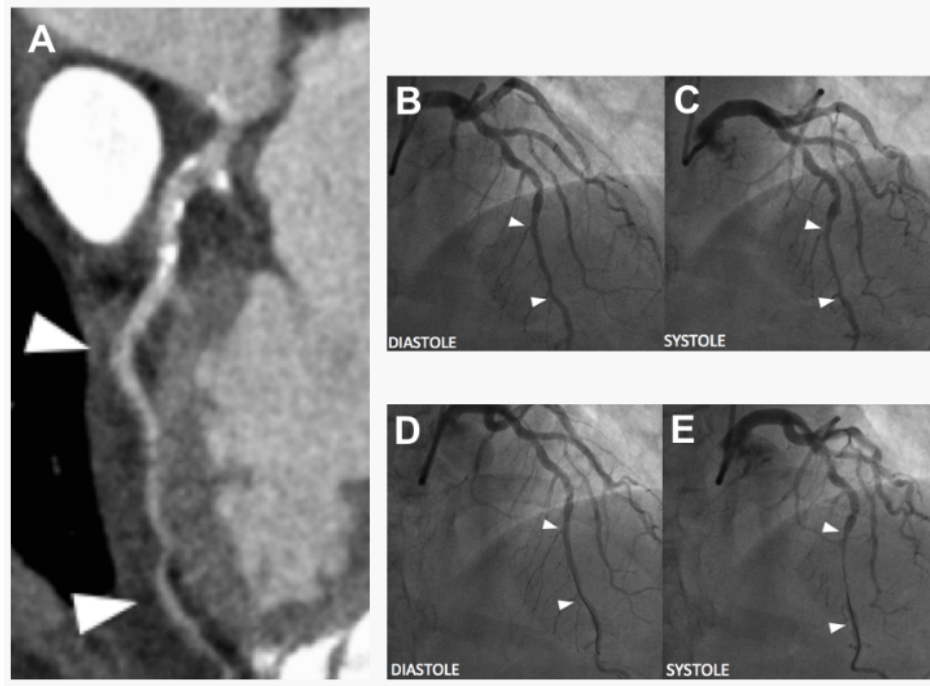

\section{BASAL}

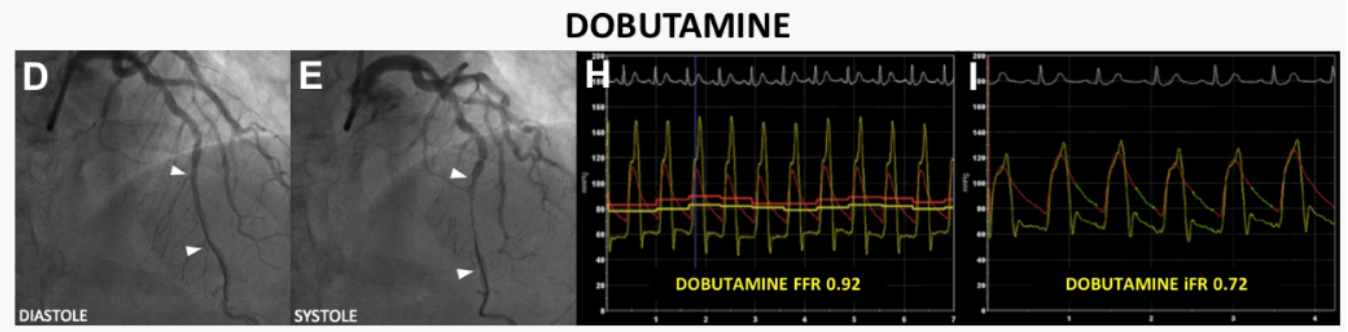

Figure 1: Diagnostic workup of a patient with left anterior descending artery (LAD) myocardial bridge (MB). A: Cardiac computed tomography scan of intramyocardial course of the LAD; note the step-down (top arrow) and step-up (bottom arrow). Angiographic appearance of the LAD MB (arrows), both in systole (B) and diastole, in basal conditions. D, E: Angiographic appearance of the same vessel (arrows), both in systole and diastole, after infusion of dobutamine $20 \mu / \mathrm{kg} / \mathrm{min}$. Fractional flow reserve (FFR) measurements are negative $(>0.80)$ before $(F)$ and after $(G)$ dobutamine infusion, whereas diastolic-specific indexes are positive at rest (instantaneous wave-free ratio (iFR) )H), and after dobutamine challenge (hyperemic wave-free period pressure ratio) (J).

Among noninvasive imaging tests to evaluate functional ischemic potential of MB are stress echocardiography, stress cardiac magnetic resonance, single-photon emission computed tomography and positron emission tomography ${ }^{26}$. More recently, new post-processing algorithms are available for the derivation of functional information from the anatomic assessment provided by CCT, such as transluminal attenuation gradient and computed tomography (CT)-derived FFR ${ }^{27,28}$.

Treatment of patients with a MB should be based on the presence of clinical symptoms and/or objective signs of ischemia. Symptomatic patients seem to respond well to pharmacological therapy, which appears to be the treatment of choice for the vast majority of patients ${ }^{29,30}$. Medical treatment should focus on relieving potential triggers and hemodynamic disturbances that aggravate the $\mathrm{MB}$, such as hypertension/hypertrophy, increased heart rate, reduced diastolic coronary filling period, and inappropriate compression of the coronary artery. Accordingly, b-blockers or calcium-channel antagonists are considered first-line therapy. Ivabradine can be considered in patients with contraindication to betablockers or in subjects not at heart rate target (i.e.>70 $\mathrm{bpm})^{30}$. Pure vasodilators, such as nitroglycerin, are not indicated because they can worsen symptoms due to their unfavorable influence on MB hemodynamic ${ }^{18}$.

Patients with symptoms refractory to medical therapy can be considered for percutaneous or surgical treatment, which include stent implantation, surgical myotomy and coronary artery bypass grafting (CABG). However, stent implantation in MB is limited due to concerns about higher reported rates of complications, including vessel perforation during stent deployment ${ }^{31}$, stent fracture ${ }^{32}$, instent restenosis ${ }^{33}$ and stent thrombosis ${ }^{34}$. Surgical options for $\mathrm{MB}$ are more invasive. Potential complications of myotomy include wall perforation, particularly in the case of a deep subendocardial course, ventricular aneurysm formation, and post-operative bleeding ${ }^{35}$. CABG appears to be safe and effective in very selected cases of severely symptomatic patients refractory to other therapies with extensive ( $>25 \mathrm{~mm}$ ) or deep ( $>5 \mathrm{~mm}$ ) MB ${ }^{36,37}$.

\section{Conflict of Interest}

Prof Giuseppe Tarantini received lecture fees from St. Jude Medical and Volzano. All other authors have nothing to disclose

\section{References}

1. Çiçek D, Kalay N, Müderrisoğlu H. Incidence, clinical characteristics, and 4-year follow-up of patients with isolated myocardial bridge: a retrospective, single-center, epidemiologic, coronary arteriographic follow-up study in southern Turkey. Cardiovasc Revascularization Med [Internet]. 2011 Jan [cited 2018 Jun 24]; 12(1): 25-8. Available from: http://linkinghub.elsevier.com/retrieve/pii/S1553838910000072

2. Möhlenkamp S, Hort W, Ge J, et al. Update on myocardial bridging. Circulation [Internet]. 2002 Nov 12 [cited 2018 Jun 24]; 106(20): 2616-22. Available from: http://www.ncbi.nlm.nih.gov/ pubmed/12427660

3. Rossi L, Dander B, Nidasio GP, et al. Myocardial bridges and ischemic heart disease. Eur Heart J [Internet]. 1980 Aug [cited 2018 Jun 24]; 1(4): 239-45. Available from: http://www.ncbi.nlm.nih.gov/ pubmed/7274234

4. Lee MS, Chen CH. Myocardial Bridging: An Up-to-Date Review. J 
Invasive Cardiol [Internet]. 2015 Nov [cited 2018 Jun 24]; 27(11): 521 8. Available from: http://www.ncbi.nlm.nih.gov/pubmed/25999138

5. Migliore F, Maffei E, Perazzolo Marra M, et al. LAD coronary artery myocardial bridging and apical ballooning syndrome. JACC Cardiovasc Imaging. 2013 Jan; 6(1): 32-41.

6. Migliore F, Zorzi A, Marra MP, et al. Myocardial edema underlies dynamic T-wave inversion (Wellens' ECG pattern) in patients with reversible left ventricular dysfunction. Hear Rhythm [Internet]. 2011 Oct [cited 2018 Jun 24]; 8(10): 1629-34. Available from: http:// linkinghub.elsevier.com/retrieve/pii/S1547527111005534

7. Feld H, Guadanino V, Hollander G, et al. Exercise-induced ventricular tachycardia in association with a myocardial bridge. Chest [Internet]. 1991 May [cited 2018 Jun 24]; 99(5): 1295-6. Available from: http:// www.ncbi.nlm.nih.gov/pubmed/2019202

8. Desseigne P, Tabib A, Loire R. [Myocardial bridging on the left anterior descending coronary artery and sudden death. Apropos of 19 cases with autopsy]. Arch Mal Coeur Vaiss [Internet]. 1991 Apr [cited 2018 Jun 24]; 84(4): 511-6. Available from: http://www.ncbi.nlm.nih.gov/ pubmed/2064513

9. Corban MT, Hung OY, Eshtehardi P, et al. Myocardial bridging: contemporary understanding of pathophysiology with implications for diagnostic and therapeutic strategies. J Am Coll Cardiol [Internet]. 2014 Jun 10 [cited 2018 Jun 24]; 63(22): 2346-55. Available from: http://linkinghub.elsevier.com/retrieve/pii/S0735109714011073

10. Bourassa MG, Butnaru A, Lespérance J, et al. Symptomatic myocardial bridges: overview of ischemic mechanisms and current diagnostic and treatment strategies. J Am Coll Cardiol [Internet]. 2003 Feb 5 [cited 2018 Jun 24]; 41(3): 351-9. Available from: http://www.ncbi. nlm.nih.gov/pubmed/12575960

11. Gould KL, Johnson NP. Imaging Coronary Blood Flow in AS: Let the Data Talk, Again. J Am Coll Cardiol [Internet]. 2016 Mar 29 [cited 2018 Jun 24]; 67(12): 1423-6. Available from: http://linkinghub.elsevier. com/retrieve/pii/S0735109716005982

12. Gould KL, Johnson NP. Myocardial Bridges: Lessons in Clinical Coronary Pathophysiology. JACC Cardiovasc Imaging [Internet]. 2015 Jun [cited 2018 Jun 24]; 8(6): 705-9. Available from: http:// linkinghub.elsevier.com/retrieve/pii/S1936878X15002181

13. Gould KL, Kirkeeide R, Johnson NP. Coronary branch steal: experimental validation and clinical implications of interacting stenosis in branching coronary arteries. Circ Cardiovasc Imaging [Internet]. 2010 Nov 1 [cited 2018 Jun 24]; 3(6): 701-9. Available from: http://circimaging.ahajournals.org/cgi/doi/10.1161/ CIRCIMAGING.110.937656

14. Ishikawa Y, Akasaka Y, Suzuki K, et al. Anatomic Properties of Myocardial Bridge Predisposing to Myocardial Infarction. Circulation [Internet]. 2009 Aug 4 [cited 2018 Jun 24]; 120(5): 376-83. Available from: http://www.ncbi.nlm.nih.gov/pubmed/19620504

15. Uusitalo V, Saraste A, Pietilä M, et al. The Functional Effects of Intramural Course of Coronary Arteries and its Relation to Coronary Atherosclerosis. JACC Cardiovasc Imaging [Internet]. 2015 Jun [cited 2018 Jun 24]; 8(6): 697-704. Available from: http://linkinghub. elsevier.com/retrieve/pii/S1936878X15002156

16. Wu S, Liu W, Zhou Y. Spontaneous coronary artery dissection in the presence of myocardial bridge causing myocardial infarction: an insight into mechanism. Int J Cardiol [Internet]. 2016 Mar 1 [cited 2018 Jun 24]; 206: 77-8. Available from: http://linkinghub.elsevier. com/retrieve/pii/S0167527316300936

17. Juilliére Y, Berder V, Suty-Selton C, et al. Isolated myocardial bridges with angiographic milking of the left anterior descending coronary artery: a long-term follow-up study. Am Heart J [Internet]. 1995 Apr [cited 2018 Jun 24]; 129(4): 663-5. Available from: http://www.ncbi. nlm.nih.gov/pubmed/7900614

18. Hongo $\mathrm{Y}$, Tada H, Ito $\mathrm{K}$, et al. Augmentation of vessel squeezing at coronary-myocardial bridge by nitroglycerin: study by quantitative coronary angiography and intravascular ultrasound. Am Heart J [Internet]. 1999 Aug [cited 2018 Jun 24]; 138(2 Pt 1): 345-50. Available from: http://www.ncbi.nlm.nih.gov/pubmed/10426850

19. Ge J, Erbel R, Rupprecht HJ, et al. Comparison of intravascular ultrasound and angiography in the assessment of myocardial bridging. Circulation [Internet]. 1994 Apr [cited 2018 Jun 24]; 89(4): 1725-32. Available from: http://www.ncbi.nlm.nih.gov/pubmed/8149538

20. Konen E, Goitein O, Sternik L, et al. The prevalence and anatomical patterns of intramuscular coronary arteries: a coronary computed tomography angiographic study. J Am Coll Cardiol [Internet]. 2007 Feb 6 [cited 2018 Jun 24]; 49(5): 587-93. Available from: http:// linkinghub.elsevier.com/retrieve/pii/S0735109706028543

21. Hakeem A, Cilingiroglu M, Leesar MA. Hemodynamic and intravascular ultrasound assessment of myocardial bridging: fractional flow reserve paradox with dobutamine versus adenosine. Catheter Cardiovasc Interv [Internet]. $2010 \mathrm{Feb} 1$ [cited 2018 Jun 24]; 75(2): 229-36. Available from: http://doi.wiley.com/10.1002/ccd.22237

22. Escaned J, Cortés J, Flores A, et al. Importance of diastolic fractional flow reserve and dobutamine challenge in physiologic assessment of myocardial bridging. J Am Coll Cardiol [Internet]. 2003 Jul 16 [cited 2018 Jun 24]; 42(2): 226-33. Available from: http://www.ncbi.nlm. nih.gov/pubmed/12875756

23. Sen S, Asrress KN, Nijjer S, et al. Diagnostic classification of the instantaneous wave-free ratio is equivalent to fractional flow reserve and is not improved with adenosine administration. Results of CLARIFY (Classification Accuracy of Pressure-Only Ratios Against Indices Using Flow Study). J Am Coll Cardiol [Internet]. 2013 Apr 2 [cited 2018 Jun 24]; 61(13): 1409-20. Available from: http:// linkinghub.elsevier.com/retrieve/pii/S0735109713004002

24. Tarantini G, Barioli A, Nai Fovino L, et al. Unmasking Myocardial Bridge-Related Ischemia by Intracoronary Functional Evaluation. Circ Cardiovasc Interv [Internet]. 2018 Jun 14 [cited 2018 Jun 24]; 11(6): e006247. Available from: http://circinterventions.ahajournals.org/ lookup/doi/10.1161/CIRCINTERVENTIONS.117.006247

25. Klues HG, Schwarz ER, vom Dahl J, et al. Disturbed intracoronary hemodynamics in myocardial bridging: early normalization by intracoronary stent placement. Circulation [Internet]. 1997 Nov 4 [cited 2018 Jun 24]; 96(9): 2905-13. Available from: http://www. ncbi.nlm.nih.gov/pubmed/9386156

26. Danad I, Szymonifka J, Twisk JWR, et al. Diagnostic performance of cardiac imaging methods to diagnose ischaemia-causing coronary artery disease when directly compared with fractional flow reserve as a reference standard: a meta-analysis. Eur Heart J [Internet]. 2017 May 1 [cited 2018 Jun 25]; 38(13): 991-8. Available from: http://eurheartj. oxfordjournals.org/lookup/doi/10.1093/eurheartj/ehw095

27. Stuijfzand WJ, Danad I, Raijmakers PG, et al. Additional value of transluminal attenuation gradient in $\mathrm{CT}$ angiography to predict hemodynamic significance of coronary artery stenosis. JACC Cardiovasc Imaging [Internet]. 2014 Apr [cited 2018 Jun 25]; 7(4): 374-86. Available from: http://linkinghub.elsevier.com/retrieve/pii/ S1936878X14000680

28. Yoon YE, Choi JH, Kim JH, et al. Noninvasive diagnosis of ischemiacausing coronary stenosis using CT angiography: diagnostic value of transluminal attenuation gradient and fractional flow reserve computed from coronary CT angiography compared to invasively measured fractional flow reserve. JACC Cardiovasc Imaging [Internet]. 2012 Nov [cited 2018 Jun 25]; 5(11): 1088-96. Available from: http:// linkinghub.elsevier.com/retrieve/pii/S1936878X12007589

29. Schwarz ER, Gupta R, Haager PK, et al. Myocardial bridging in absence of coronary artery disease: proposal of a new classification based on clinical-angiographic data and long-term follow-up. Cardiology [Internet]. 2009 [cited 2018 Jun 25]; 112(1): 13-21. Available from: https://www.karger.com/Article/FullText/137693 
30. Schwarz ER, Klues HG, vom Dahl J, et al. Functional, angiographic and intracoronary Doppler flow characteristics in symptomatic patients with myocardial bridging: effect of short-term intravenous betablocker medication. J Am Coll Cardiol [Internet]. 1996 Jun [cited 2018 Jun 25]; 27(7): 1637-45. Available from: http://www.ncbi.nlm.nih. gov/pubmed/8636548

31. Ernst A, Bulum J, Šeparović Hanževački J, et al. Five-year angiographic and clinical follow-up of patients with drug-eluting stent implantation for symptomatic myocardial bridging in absence of coronary atherosclerotic disease. J Invasive Cardiol [Internet]. 2013 Nov [cited 2018 Jun 25]; 25(11): 586-92. Available from: http://www.ncbi.nlm. nih.gov/pubmed/24184893

32. Srinivasan M, Prasad A. Metal fatigue in myocardial bridges: stent fracture limits the efficacy of drug-eluting stents. J Invasive Cardiol [Internet]. 2011 Jun [cited 2018 Jun 25]; 23(6): E150-2. Available from: http://www.ncbi.nlm.nih.gov/pubmed/21646663

33. Haager PK, Schwarz ER, vom Dahl J, et al. Long term angiographic and clinical follow up in patients with stent implantation for symptomatic myocardial bridging. Heart [Internet]. 2000 Oct [cited 2018 Jun 25]; 84(4): 403-8. Available from: http://www.ncbi.nlm.nih.gov/ pubmed/10995410
34. Agirbasli M, Hillegass WB, Chapman GD, et al. Stent procedure complicated by thrombus formation distal to the lesion within a muscle bridge. Cathet Cardiovasc Diagn [Internet]. 1998 Jan [cited 2018 Jun 25]; 43(1): 73-6. Available from: http://www.ncbi.nlm.nih. gov/pubmed/9473197

35. Iversen S, Hake U, Mayer E, et al. Surgical treatment of myocardial bridging causing coronary artery obstruction. Scand J Thorac Cardiovasc Surg [Internet]. 1992 [cited 2018 Jun 25]; 26(2): 107-11. Available from: http://www.ncbi.nlm.nih.gov/pubmed/1439639

36. Bockeria LA, Sukhanov SG, Orekhova EN, et al. Results of Coronary Artery Bypass Grafting in Myocardial Bridging of Left Anterior Descending Artery. J Card Surg [Internet]. 2013 May [cited 2018 Jun 25];28(3):218-21. Available from: http://www.ncbi.nlm.nih.gov/ pubmed/23574298

37. Attaran S, Moscarelli M, Athanasiou T, et al. Is coronary artery bypass grafting an acceptable alternative to myotomy for the treatment of myocardial bridging. Interact Cardiovasc Thorac Surg [Internet]. 2013 Mar 1 [cited 2018 Jun 25]; 16(3): 347-9. Available from: https://academic.oup.com/icvts/article-lookup/ doi/10.1093/icvts/ivs459 